\title{
Overcoming the barriers to the diagnosis and management of chronic fatigue syndrome/ME in primary care: a meta synthesis of qualitative studies
}

Kerin Bayliss ${ }^{1 *}$, Mark Goodall², Anna Chisholm³ , Beth Fordham³ ${ }^{3}$ Carolyn Chew-Graham ${ }^{4}$, Lisa Riste ${ }^{1}$, Louise Fisher ${ }^{5}$, Karina Lovell ${ }^{6}$, Sarah Peters ${ }^{7}$ and Alison Wearden ${ }^{7}$

\begin{abstract}
Background: The NICE guideline for Chronic Fatigue Syndrome/Myalgic Encephalomyelitis (CFS/ME) emphasises the need for an early diagnosis in primary care with management tailored to patient needs. However, GPs can be reluctant to make a diagnosis and are unsure how to manage people with the condition.

Methods: A meta synthesis of published qualitative studies was conducted, producing a multi-perspective description of barriers to the diagnosis and management of CFS/ME, and the ways that some health professionals have been able to overcome them. Analysis provided second-order interpretation of the original findings and developed third-order constructs to provide recommendations for the medical curriculum.

Results: Twenty one qualitative studies were identified. The literature shows that for over 20 years health professionals have reported a limited understanding of CFS/ME. Working within the framework of the biomedical model has also led some GPs to be sceptical about the existence of the condition. GPs who provide a diagnosis tend to have a broader, multifactorial, model of the condition and more positive attitudes towards CFS/ME. These GPs collaborate with patients to reach agreement on symptom management, and use their therapeutic skills to promote self care.

Conclusions: In order to address barriers to the diagnosis and management of CFS/ME in primary care, the limitations of the biomedical model needs to be recognised. A more flexible bio-psychosocial approach is recommended where medical school training aims to equip practitioners with the skills needed to understand, support and manage patients and provide a pathway to refer for specialist input.
\end{abstract}

Keywords: Chronic fatigue syndrome/ME, Barriers and facilitators, Management and diagnosis, Qualitative research, Primary health care

\section{Background}

Chronic Fatigue Syndrome (CFS) or Myalgic Encephalomyelitis (ME) is characterised by disabling, unexplained fatigue that is not alleviated by rest and lasts at least four months [1]. Symptoms can include headaches, unrefreshing sleep, pain, sore throat, concentration or memory problems and post exertional malaise [1]. The diagnosis is made after all relevant differential diagnoses have been

\footnotetext{
*Correspondence: kerin.bayliss@manchester.ac.uk

'Institute of Population Health, University of Manchester, Manchester, UK Full list of author information is available at the end of the article
}

excluded; the prevalence of CFS/ME among adults in both the US and the UK is estimated at around $0.2-0.4 \%$ [2]. The condition is distressing and costly in terms of both health service utilization and economic burden to patients and their families $[3,4]$.

The UK National Institute for Health and Clinical Excellence (NICE) guideline, published in 2007 for CFS/ $\mathrm{ME}$, emphasises the importance of an early diagnosis [1]. However, many patients continue to experience the same numerous, complex barriers to diagnosis that were described in the 1990s [5-9]. For example, in a recent 
treatment trial, primary care patients reported waiting, on average, 3.7 years from onset of symptoms to diagnosis [10]. NICE guidance also recommends that patients with CFS/ME receive early treatment with the use of tailored care-packages [1]. However, $65 \%$ of members of a UK patient organisation, Action for ME, reported never receiving any treatment [11].

One possible reason for the lack of progress in the diagnosis and management of CFS/ME, is that training at medical school can engender negative attitudes and a lack of confidence in the management of CFS/ME [12]. The low status of CFS/ME is reinforced in UK practice as it is not incentivised as part of the Quality and Outcome Framework, a pay-for-performance scheme that financially rewards GP practices for achieving a number of clinical indicators [13,14].

This paper presents a meta synthesis of published qualitative studies that provide rich, bottom up data on the barriers to the diagnosis and management of CFS/ $\mathrm{ME}$, and the ways that some health professionals have overcome them. As the aim of the paper is to explore the experiences, values and behaviours of patients and health professionals, the authors have chosen not to include quantitative studies. This is because quantitative data does not provide sufficient insight into the reasons why some GPs are able to successfully manage CFS/ME while others are not, or the factors that help or hinder GPs within a consultation. The qualitative literature also explores issues that are external to the consultation such as policy development, taking a multifactoral approach to understanding the process of managing CFS/ME in primary care $[15,16]$. This paper will examine commonalities between these qualitative studies in order to make recommendations for the development of the UK medical curriculum, with the aim to improve care.

\section{Research question}

What recommendations can be made for the development of the UK medical curriculum, the training of GPs and members of other health care professions, in order to overcome the barriers to the diagnosis and management of CFS/ME in primary care?

\section{Methods}

Qualitative meta synthesis was used to identify interrelated themes from relevant published qualitative studies (Stages of the meta synthesis) $[17,18]$.

\section{Stages of the meta synthesis}

1. Identifying the literature: topic selection, searching for studies, appraisal of studies.

2. Data analysis and interpretation: a. extraction of main findings from the published studies,

b. synthesis of main findings into themes to form an explanatory framework.

\section{Identifying the literature}

The focus of our work was based on two substantive areas. The first was the major themes and findings related to the barriers to diagnosis and management of CFS/ME, and the second was the implications and recommendations for overcoming these barriers. A literature search was performed using Medline/PubMed, PsycINFO, CINAHL and Web of Science databases. Our search keywords were Chronic Fatigue Syndrome or CFS or Myalgic Encephalomyelitis or Myalgic Encephalitis, which were combined, using Boolean logic terms "or" and "and", with the following list of search terms: doctor; family physician; family practice; general practice; General Practitioner; GP; and Primary Care; service. We limited our search to published English language articles and to the last 30 years of publications. The search terms, inclusion criteria and the procedure for generation of the final sample of studies are displayed in Figure 1.

After removal of duplicates, the database searches produced 496 abstracts, dated from 1988 to 2013. All abstracts were screened using the following initial questions: Is this qualitative research?; Is this research based within a primary care setting?; Does it look at patient's beliefs and views on the diagnosis, and management of CFS/ME?; Does it examine the practitioner's perceptions of the diagnosis, management of CFS/ME?. This produced a subset of 47 articles which were reviewed by AW and AC. Consequently, 26 articles were excluded, which although they examined the process diagnosis and management of CFS/ ME within primary care, they did not report the views of either patients or practitioner to this process. In total, 21 qualitative articles were included (Figure 1). Table 1 details the characteristics of these articles.

\section{Quality appraisal}

Guided by an existing quality appraisal framework that used a modified version of the CASP (Critical Appraisal Skills Programme) qualitative checklist, [38] a relevant quality appraisal tool was created by the authors and tailored to meet the aims of the meta synthesis (Appendix 1). Each paper was rated on the following criteria, using a three point scale $(0=$ Serious methodological issues; $1=$ Minor methodological issues; 2 = Robust):

1) how relevant was the paper was to the present study's research question?

2) to what extent did the paper add value to answering the research question?

3) and how methodologically robust was the study? 




Figure 1 Flow diagram of search strategy and article selection.

These ratings were then combined to categorise each paper as "key", "adequate and relevant" or "flawed or not relevant" (Appendix 1). The aim was to remove any papers that were rated "flawed or not relevant" from our analysis.

Two researchers (AC and BF) carried out the quality appraisals. The researchers independently coded the remaining papers using the above criteria, thereby ensuring that all papers were double coded. Initial disagreements were successfully resolved via discussion and agreement was reached on all ratings. For example, it was highlighted that there was ambiguity around how to rate 'relevance' when a paper's explicit research aim did not match the present study's research question. Following discussion it was decided that if the findings explicitly identified ideas and concepts that answered the present study's research question, that paper would not be penalised regarding its relevance. Furthermore it was decided that papers receiving the highest possible rating ( 2 for each criteria) would be categorised as key papers (Table 1). None of the 21 papers identified for analysis were classed as "not relevant".

\section{Data analysis and interpretation}

$\mathrm{KB}, \mathrm{AW}$ and MG read and analysed each of the articles. A grid was constructed with studies along the $\mathrm{x}$-axis and the content components (essential findings and interpretations) along the y-axis. Data was extracted on the original author's analysis of the primary qualitative data. The themes derived from this primary data are called first order constructs $[17,18]$. A thematic approach was then taken, grouping first order constructs from each paper into core themes. The researchers recorded which papers contributed to each theme, in terms of relevant data or contradictory or contrasting results. The analysis was completed independently and then the authors met to discuss, examine and agree on emergent themes. The final core themes are termed second-order constructs as they emerge from the analysis of first order analysis of the primary data. The recommendations for practice in this meta analysis are not only consistent with the original results but also extend beyond them, considering how the second order constructs sit within the wider literature. These recommendations for the medical curriculum are termed third order constructs $[17,18]$. 
Table 1 Overview of the included studies

\begin{tabular}{|c|c|c|c|c|c|}
\hline Study ID & Population (N) & $\begin{array}{l}\text { Country } \\
\text { setting }\end{array}$ & Primary objectives & Methods, recruitment and analysis & $\begin{array}{l}\text { Appraisal of quality } \\
\text { and relevance }\end{array}$ \\
\hline \multirow{3}{*}{$\begin{array}{l}\text { Asbring and } \\
\text { Narvanen [19] }\end{array}$} & \multirow[t]{3}{*}{26 health professionals } & \multirow[t]{3}{*}{ Sweden } & \multirow{3}{*}{$\begin{array}{l}\text { Explore physicians' perspectives on CFS and } \\
\text { fibromyalgia patients, specifically their thoughts } \\
\text { about these patient groups and what strategies } \\
\text { they use in consultations with them. }\end{array}$} & Methods: Semi-structured interviews & \multirow[t]{3}{*}{ Relevant: 2-2-1 } \\
\hline & & & & $\begin{array}{l}\text { Recruitment: CFS/fibromyalgia patients } \\
\text { contacted and asked to identify physicians } \\
\text { (patients identified through a previous study) }\end{array}$ & \\
\hline & & & & $\begin{array}{l}\text { Analysis: Grounded theory principles (including } \\
\text { constant comparison and thematic saturation } \\
\text { but not theory development) }\end{array}$ & \\
\hline \multirow[t]{3}{*}{ Ax et al. [20] } & \multirow{3}{*}{$\begin{array}{l}18 \text { (9 patients each in } 2 \\
\text { studies) }\end{array}$} & \multirow[t]{3}{*}{ UK (England) } & \multirow{3}{*}{$\begin{array}{l}\text { Explore CFS sufferers' accounts of patient- } \\
\text { professional communication, patient illness } \\
\text { beliefs and treatment expectations, and } \\
\text { consequences of interactions regarding } \\
\text { treatment choice. }\end{array}$} & Methods: Semi-structured interviews & \multirow[t]{3}{*}{ Relevant: 2-1-1 } \\
\hline & & & & Recruitment: ME support group invitation & \\
\hline & & & & Analysis: Content analysis & \\
\hline Banks and Prior & 114 consultations observed & UK (Wales) & Investigate lay and professional ideas about the & Methods: Patient-professional observations in an & Relevant: 2-2-1 \\
\hline
\end{tabular}

$\begin{array}{ll}\text { Banks and Prior } & 114 \text { consultations observed } \\ {[21]} & \text { with patients and health } \\ \text { professionals. }\end{array}$

Bayliss et al. [22] 35 (11 patients, 2 carers, 9 GPs, 5 practice nurses, 4 CFS/ME

specialists and $5 \mathrm{BME}$ community leaders)

Chew-Graham et al. [7]
38 (24 patients; 14 physicians)

UK (England)

Explore practice nurses' beliefs about CFS patients and their perceived role regarding management.

UK (England)

Explore how CFS patients and physicians the clinical consultation. nvestigate lay and professional ideas about the nature of CFS, in

particular, the ways in which understandings of the disorder are developed in a clinical setting.

Explore BME patient, health professional and community leader's views on the barriers to the diagnosis and management of CFS/ME in the

BME population understand the condition and how this affects

Fplore GP' views on their role in diagnosing and managing CFS patients.
Methods: Patient-professional observations in out-patients clinic, and structured interviews with patients.

Recruitment: CFS clinic (no further details)

Analysis: No clear description. Authors took a functional approach and analysed accounts of illness rather than beliefs about illness.

Methods: Semi-structured interviews

Key: 2-2-2

Recruitment: Invited by letter/phone.

Analysis: Thematic analysis

Methods: Semi-structured interviews

Recruitment: Purposive sampling from participants within a previous study (physicians nominated eligible patients).

Analysis: Thematic analysis using constant comparison principles

Methods: Semi-structured interviews

Recruitment: Identified via involvement with a previous study and invited by letter/phone.

Analysis: Thematic analysis

Methods: Semi-structured interviews

Relevant: 2-2-1

Recruitment: Identified via involvement with a previous study and invited by letter/phone.

Analysis: Thematic analysis 
Table 1 Overview of the included studies (Continued)

Chew-Graham 19 patients UK (England)

9 patients

UK (England)

Establish important factors for patients

engaging in a CFS intervention and make

Methods: Semi-structured interviews

Key: $2-2-2$

recommendations for GP on referring patients

to such a service.

Clarke [26]

60 patients

Canada

Describe the way in which CFS patients seek confirmation and legitimisation of their illness.

Explore patients' experiences of living with CFS.

tment: Identified GPs within a previous study and asked them to refer registered CFS

patients to the study.

Analysis: Thematic analysis

Methods: Open-ended focused interviews

Relevant: 2-2-1

Recruitment: Patients contacted through CFS support groups and invited to participate via etter.

Analysis: Cross-case analysis

Methods: Interviews

Recruitment: Patients contacted through alternative therapy clinics (Reiki) or persona contacts.

Analysis: Interpretative Phenomenological Analysis (IPA)

Methods: Semi-structured interviews

Explore the experiences of living with CFS to increase insight into the experiences of and difficulties faced by people with this condition.

Recruitment: Members of an ME self-help network were recruited via posters and email.

Analysis: IPA

Methods: Group interview

Explore obstructions for quality care experienced by people with CFS

Recruitment: Purposive sampling from patient organisation.

Analysis: Systematic text condensation (Giorgi, 1985)

Method: Non-directive discussion topic in an online forum.

professionals and patients with CFS. Targeting sensitive issues in an online environment and exploring how the accounts were constructed.

Recruitment: Patient support group invitation Analysis: Discursive analysis

Methods: Semi-structured interviews

Explore patient, carer and health professional's

44 (9 GPs, 5 Practice Nurses, CFS/ME specialists, 10 carers and 16 patients)

UK (England) views on the development of CFS/ME training and resources for primary care.

Recruitment: Invited by letter/phone.

Analysis: Thematic analysis.

Method: Semi-structure interview with developed topic guide

Recruitment: Nominated by members of England-wide study.

Analysis: Thematic analysis. 
Table 1 Overview of the included studies (Continued)

Horton-Salway 10 GPs UK (England

[32]

10 GPs

K (England)

Explore GP's construction of CFS/ME patient

identities and the definition of their illness.

Method: Unstructured broad theme one-to-one

Relevant: 2-2-1

interviews.

Recruitment: Nominated by members of a

patient support group

Analysis: Discourse analysis.

$\begin{array}{lll}\text { McDermott et al. } 20 \text { patients } & \text { UK (England) } & \text { Explore hopes and expectations of patients } \\ & \text { newly referred to CFS service (Department of } \\ & \text { Health/National Institute of Health and Clinical } \\ & \text { Excellence). }\end{array}$

Method: Semi-structure interview with

developed topic guide

Key: 2-2-2

Recruitment: Invitation letter sent to patients newly referred to specialist CFS service by their GP.

Analysis: Constant comparative analysis.

Peters et al. [34] 46 patients, 3 nurses and 2 supervisors

UK (England)

Identify potential barriers and solutions for general nurse practitioners in implementing

psychosocial interventions to people with CFS. Taken from 3 perspectives (the nurses delivering the intervention, the patients and supervisors).

Raine et al. [35] $\quad 46$ GPs

UK (England)

Explore GPs perspective about CFS and irritable bowel symptoms and how they should be treated.

Expand upon quantitative quality of life measurements to understand how healthcare (diagnosis and management) impacts upon quality of life for people with CFS and Fibromyalgia.
Compare GP and patients perspective of CFS and its management
Method: Mixed methods nested qualitative

study. Semi-structured interview with developed

topic guid

Recruitment: Purposive and matched sampling

(age etc)

Analysis: Thematic analysis.

Method: Nominal groups (clinical guideline opinion groups). Scenario evaluation.

Recruitment: Random sample

Analysis: Grounded theory variant.

Method: Mixed method design. Semi-structure telephone interview.

Recruitment: Convenience sample from 3 loca support groups.

Analysis: Constant comparative analysis.

Method: Semi-structure interview with

developed topic guide

Recruitment: GPs recruited from Royal College.

Unclear how patients were recruited.

Analysis: No analytic procedure defined. Mixed quantitative e.g. $50 \%$ of doctors believed..." 


\section{Results}

Table 1 provides an overview of the study population, the methodological strategies and the appraisal of relevance and robustness of the research. The 21 studies included in the meta-synthesis were conducted in the UK (16 studies), USA, Canada, Australia, Sweden and Norway.

The synthesis of themes is presented in two sections. The first section includes four themes which describe the barriers to the diagnosis and management of CFS/ $\mathrm{ME}$, and the second section outlines three themes that relate to overcoming these barriers.

\section{Themes}

1. Barriers to the diagnosis and management of CFS/ $\mathrm{ME}$

1.1 Illness models

1.2 The health professional-patient relationship

1.3 Knowledge and attitudes

1.4 Priorities in primary care

2. Overcoming barriers

2.1 Developing positive attitudes to CFS/ME

2.2 Developing therapeutic skills

2.3 Taking a collaborative approach

\section{Barriers to the diagnosis and management of CFS/ME Illness models}

The literature shows that both health professionals and patients tend to take a predominantly biomedical approach to understanding illness. This approach holds that symptoms are caused by underlying disease that can be measured by a definitive diagnostic test [19,22-24,26,31]. In the case of CFS/ME, the biomedical approach, which is central to the medical curriculum, leads many health professionals to conclude that there is no real illness as there is currently no identifiable pathology [7,20,23-26,31,35] while patients conclude that there must be a disease because they know there is an illness [7,21,23,31,35,38]. Despite patients experiencing real, disabling and chronic symptoms, a key paper in our analysis highlights that the scepticism among health professionals about the status of CFS/ME can lead to reluctance to make a diagnosis [7].

In primary care, it has been reported that some GPs provide a psychological label such as depression in order to avoid saying that their diagnosis is uncertain [20,22,29,31]. Other health professionals hold a "somatisation" model of illness where patients are thought to be expressing social and emotional problems in physical symptoms [19,32,37]. This approach can be experienced by patients as a blame shifting device with patients feeling held accountable for their poor health [32]. Patients may equate an interpretation of symptoms as having an origin in psychological or social problems with a belief that symptoms are imagined or fictitious and therefore reject this approach $[19,20,29,31,34]$.
It is important for patients to feel that their symptoms are accepted and believed in order for them to engage in the management of their CFS/ME $[25,27,32,34,36]$. If patients are faced with a conflicting illness model, and ongoing disbelief as to the reality of their condition, they may disengage with primary care [22,29,31].

\section{The health professional-patient relationship}

Studies have highlighted poor communication between the patient and the health professional as a barrier to diagnosis and management of CFS/ME [7,37]. For example, GPs can fail to validate the patients' illness experience or explain the rationale for treatment [7]. Health professionals can also be confused by the way some patients present their story [7]. Tensions are created when patients have experienced unsatisfactory consultations with previous GPs who have not provided a diagnosis. This can lead patients to be defensive or to present complicated stories with emotion or frustration [29]. Rushed consultations can also mean that patients feel unable to communicate the full extent or context of their condition [7].

Asbring and Narvanen, [19] describe how GPs can experience a lack of control in the consultation and the inability to provide a biomedical diagnosis and prescribe medication can lead to a sense of powerlessness and frustration. If a patient does not improve, practice nurses and GPs also report a lack of job satisfaction, and can distance themselves from the patient [19,23]. The breakdown of the GP-patient relationship can lead to a lack of empathetic care and patients can feel helpless and let down $[7,19,28,29,35]$.

Bayliss et al. [22] describe how Black and Minority Ethnic (BME) patients who do not have English as their first language are not able to adequately describe their symptoms or understand the GP during a consultation. As a diagnosis of CFS/ME requires other conditions to be excluded, a number of appointments and investigations are required to reach diagnosis, which is made more difficult when communication is difficult. The term 'Chronic Fatigue Syndrome' can also be difficult to understand in these patient groups [22].

A poor GP-patient relationship can mean that patients turn to support groups rather than primary care for information and support. Patients with experience of CFS/ $\mathrm{ME}$ can act as counsellors to new sufferers $[20,26]$. Some patients also use complementary therapy or identify types of 'self help' [28]. The choice of therapy was often based on recommendations from support groups $[20,26]$.

\section{Knowledge and attitudes}

Raine et al. [35] report that GPs can act on their often limited understanding of CFS/ME, with little insight into what it means for the patient. Some GPs felt that their medical education had failed to equip them with the 
therapeutic skills necessary for diagnosing and managing patients with CFS/ME in the way recommended by current guidelines $[7,19,24,27,29,33,37]$. Training can also be coloured by personal opinions that senior staff hold, with patients with CFS/ME sometimes being portrayed in a derogatory manner [23,37]. A lack of knowledge about specialist services was also highlighted in the literature; with some GPs not knowing what interventions are available [25,31,38]. Patients also believed that GPs lacked the time or therapeutic expertise required to provide a sufficient level of emotional support $[20,33,36]$.

The literature shows that some health professionals can hold inappropriately negative attitudes and stereotypical views of CFS/ME that can act as a barrier to diagnosis. For example, a number of studies have shown that some GPs believe that there are certain types of people who get CFS/ME, including those who have hypochondriasis, [36] are unmotivated, [30] pessimistic or are difficult to help $[19,22,29,35]$. These health professionals can believe that a diagnosis has little constructive value to patients as there are no pharmacological treatments, and that diagnosis might even become a disabling self-fulfilling prophecy $[23,24,26,37]$. The fact that some patients, while suffering from severe and disabling symptoms, may not look sick, can contribute to inaccurate perceptions of the condition [19].

\section{Priorities in primary care}

Asbring and Narvanen [19], writing in the Swedish context, suggested that CFS/ME is low ranking in the medical hierarchy as symptoms are not life threatening and it may be believed that it cannot be cured $[19,29]$. In the UK, health professionals also believe that there are insufficient patients with CFS/ME within their registered practice population to justify the investment of time and resources in developing and maintaining appropriate expertise [23]. Practice nurses claimed that they would be unable to develop a role in the management of patients with CFS/ME unless practices would financially benefit from this work [23]. The low status of CFS/ME in primary care is reflected in patient interviews where many describe themselves as experiencing limited medical care and attention [30].

\section{Overcoming the barriers}

The literature shows that some health professionals have successfully diagnosed and managed CFS/ME by taking a more flexible, bio-psychosocial approach, building a positive, collaborative, therapeutic relationship with their patient. These lessons should inform the development of the medical curriculum in order to reduce inconsistencies in care.

\section{Developing a more positive attitude towards CFS/ME}

Health professionals have been found to change their attitude towards CFS/ME following personal experience with the condition or if they know someone with it $[7,31]$. Personal experience challenges stereotypical and sceptical views, and provides understanding of the impact of the condition on quality of life. GPs are then able to offer support, and may seek the knowledge required to provide a diagnosis and encourage symptom management $[26,28]$. This finding illustrates that attitudes can be flexible. Therefore medical school training could be more effective in engendering more positive attitudes towards the condition [5,29].

\section{Developing therapeutic skills}

GPs who diagnose CFS/ME placed particular importance on building a therapeutic relationship with the patient based on listening skills, respect and trust [31]. In order to provide the emotional support and information valued by patients, extended consultation times were required $[5,19,37]$. This allows the GP to work with the patient to prioritise the symptoms they wanted to address in order to work towards recovery [5]. Patients valued this approach, rating these GPs as particularly helpful and effective.

\section{Taking a collaborative approach}

GPs who diagnose CFS/ME adjust their own expectations and demands about what a physician can achieve, affirming the patients' accounts and learning from them $[20,25]$. Improvements may be slow and patients can experience relapse so GPs must develop resilience and understand that there is no quick fix [20]. The use of patient resources such as information sheets on how to manage the symptoms of CFS/ME can be used as a base to work with the patient to support self management [5]. Nurses also recognised the importance of investing time into explaining the rationale for the treatment and listening to and validating patients' illness experience [34]. In doing so, they reported that they were less likely to be viewed as confronting the model of illness held by the patient, and more likely to be viewed as encouraging patients to be more active in addressing their own symptoms [31,34]. This collaborative approach is therefore beneficial in the long term management of CFS/ME as it improves communication [7]. Furthermore, successful GPs recognised that the family or significant others are instrumental in helping or hindering management [22,28]. Health professionals should therefore engage with family members where possible, as without family support, patients can feel isolated and may struggle to manage their symptoms [22,27]. 


\section{Discussion}

This meta synthesis highlights a number of ways that GPs have overcome the barriers to the diagnosis and management of CFS/ME in primary care. GPs who take a therapeutic and collaborative approach are able to diagnose and manage CFS/ME in a positive way, as recommended by the UK NICE guideline $[1,7,27,31]$. This information is valuable in the development of the medical school curriculae and GP and health professional training to challenge the barriers that have led to the same inconsistencies in care for patients since the 1990s.

\section{Comparison with previous literature}

In line with the findings of a review completed by Elliot [39] in the 1990s, our analysis suggests that the reluctance to diagnose and manage CFS/ME is based on scepticism and a lack of knowledge about the condition [7,23, 26,31,40,41]. Edwards et al. [28] reports that a lack of training at medical school on conditions commonly called "medically unexplained" can cause frustration in the patient which leads to a breakdown of the GP-patient relationship. Larun and Malterud [15] also highlight that a diagnosis and information on the condition was necessary for recovery, as without this, the patient can feel severely ill, yet blamed and dismissed and therefore disengage. This was also a theme in the current review which found that without a functioning relationship, communication breaks down and the GP is unable to learn from the patient's experiences or form a therapeutic relationship to manage symptoms [31]. Patients are seen as difficult and uncooperative which compounds the negative attitudes and stereotypical beliefs held by GPs about this group. Stenhoff et al., [12] describe how such negative beliefs are then passed on to medical students, creating a new generation of doctors with the same stereotypical views about patients with CFS/ME. This maintains the barriers to the diagnosis and management of CFS/ME that have existed for the past 20 years [12].

\section{Implications for practice}

The barriers to the diagnosis and management of CFS/ ME highlighted in this study result in a significant burden of dissatisfaction for both patients and health professionals $[5,24,42,43]$. However, at least in the UK, the expectation that CFS/ME will normally be diagnosed and managed within primary care means that GPs need to find a way to engage with these patients [5].

This meta synthesis highlights that only a minority of health professionals report that they are able to successfully diagnose and manage patients with CFS/ME in primary care. This finding suggests that there is a need to address the inadequacy of medical training about this condition $[5,10,15,19,23,24,31,35]$. For example, the somatisation model, used by many GPs to explain the symptoms of
CFS/ME, arises out of the failure of the biomedical model $[19,32,37]$. It comes from reasoning that if there is no disease underlying the illness, the illness must be the manifestation of emotional distress. The bio-psychosocial model may be a better starting point for explaining and teaching medical students about conditions such as CFS/ ME. This approach suggests a multi-factorial explanation for the condition, with interactions between biological, psychological and social factors maintaining symptoms. It focuses on a patient-centred consultation, using the collaborative and therapeutic skills shown to be valuable in this meta synthesis, to develop rapport and trust with a patient, listening and validating their experiences. Once the patient feels understood the GP can then go on to discuss the management of the condition [44]. CFS/ME therefore needs to be presented to students in a positive way, with clear and simple messages [45,46]. This could mean teaching students about these conditions first before they start to habitually use the biomedical model as a prototype for all conditions. Stenhoff et al. [12] state that the first step in achieving this change is to address potential negative attitudes among trainers as sceptical attitudes towards CFS/ME can be learned by students.

An additional problem in the diagnosis and management of CFS/ME is the persistence of the term "medically unexplained illness", which indicates diagnoses can only be by exclusion and does not allow for a positive diagnosis. This can provide a reason for medical professionals to delay diagnosis and even opt out of treatment as they feel that they have nothing to offer [47]. A fundamental shift in the perceived role of the GP is required to enable the holistic and therapeutic approach necessary to diagnose and manage CFS/ME in a positive way, as recommended by the NICE guideline [1].

\section{Limitations}

The aim of this study was to systematically describe other interpretative studies. However, the varying research designs and level of detail provided in the qualitative studies (Table 1) make it difficult to objectively synthesise results across every study. The synthesis also only found studies from developed countries (UK, USA, Canada, Australia, Sweden and Norway), which used convenience sampling in primary care settings, CFS/ME support groups, and patient organizations. It is possible that there are other relevant studies that were not identified by our search terms. In order to address issues of consistency of reporting for this metasynthesis, the authors focused on peer reviewed, published papers only. The grey literature was therefore not included.

Formal validation of the novel quality appraisal tool used was not conducted which may have led to bias within quality coding judgements, However, double coding procedures were used to enhance the reliability of coder 
judgements thereby reducing any subjectivity which had potential to influence the results.

A number of the authors of this paper were involved in some of the original studies included in the synthesis. This is an inevitable finding from the limited number of researchers working in the field. In order to reduce bias, four researchers who were not authors of any of the original studies were involved in the reviewing, data extraction and analysis stages.

\section{Conclusions}

This meta synthesis shows that some GPs have overcome the multiple barriers to the diagnosis and management of CFS/ME by building a positive, collaborative relationship with the patient, taking time to explain the rationale for treatment and validating the patients' illness experience. However, our analysis highlights that this good practice has not been adopted by all health professionals, and the same barriers to diagnosis and management reported in the 1990s continue to be a problem today.

In order to learn from the success stories, the literature suggests the need to broaden the understanding of what the practice of medicine entails. This includes developing the curriculum to help health professionals to consider that the biomedical model, in which illness is understood in terms of underlying disease, is not appropriate for all conditions, and to understand that patients may experience real, disabling and chronic symptoms without an identifiable underlying pathology. We suggest that by teaching a more flexible, biopsychosocial approach to understanding illness, coupled with a focus on therapeutic skills to support symptom management, medical trainers may ultimately produce practitioners who are better able to diagnose, engage with and manage patients with CFS/ME, and also understand when to refer for specialist management.

\section{Appendix 1}

CFS Meta-synthesis Quality Appraisal Form

Notes for researchers. For each article answer the questions below and make notes illustrating evidence from articles to support your judgements. Following this, assign each article a global category based on these responses. Categories are hierarchical and include the following:

Key papers: receive a score of 2 for all questions (i.e. 2-2-2).

Adequate and relevant papers: receive a score of 2 or 1 for any question (i.e. 2-2-1, 1-2-1 etc.).

Flawed or not relevant papers: receive a score of 0 for any question (i.e. 0-1-1).

Question 1: Relevance

How relevant is the article in relation to the review's research question? (Consider to what extent the article is able to address the research question and how much of the article is focused upon this)

$0=$ Not at all relevant

$1=$ Somewhat relevant

$2=$ Very relevant

Evidence from article:

Question 2: Value

What is the value of the article in terms of contributing to addressing the research question? (Consider how insightful the findings are to this field of research; and how novel/original/detailed the findings are)

$0=$ Contributes no/very little value

$1=$ Contributes some value

2 = Contributes a lot of value

Evidence from article:

Question 3: Methodological robustness

How methodologically robust is the study? (Consider suitability of research design/analysis; depth of analysis; potential for bias)

$0=$ Serious methodological issues

$1=$ Some methodological issues

$2=$ Methodologically robust

Evidence from article:

\section{Competing interests}

The authors declare that they have no competing interests.

\section{Authors' contributions}

$\mathrm{KB}$ contributed to the literature search, read and analysed each of the articles and drafted the paper. MG contributed to the literature search and read and analysed each of the articles. AC and BF completed the quality appraisal and contributed to the writing of the paper. CCG, LR, LF, KL and SP contributed to the research design and the writing the paper. AW designed and managed this meta synthesis. She contributed to the literature search, quality appraisal, analysis and writing of the paper. All authors read and approved the final manuscript.

\section{Acknowledgements}

This paper outlines independent research funded by the National Institute for Health Research (NIHR) under its Research for Patient Benefit Programme (Grant Reference PB-PG-0609-19108). The views expressed are those of the authors and not necessarily those of the NHS, the NIHR or the Department of Health.

\section{Author details}

'Institute of Population Health, University of Manchester, Manchester, UK. Institute of Psychology, Health and Society, University of Liverpool, Liverpool, UK. ${ }^{3}$ Institute of Inflammation and Repair, University of Manchester, Manchester, UK. ${ }^{4}$ Primary Care and Health Sciences and National School for Primary Care Research, Keele University, Keele, UK. ${ }^{5}$ National School for Primary Care Research, University of Manchester, Manchester, UK. ${ }^{6}$ School of Nursing, Midwifery and Social Work, University of Manchester, Manchester, UK. ${ }^{7}$ School of Psychological Sciences, University of Manchester, Manchester, UK.

Received: 8 October 2013 Accepted: 18 February 2014

Published: 7 March 2014

\section{References}

1. National Institute for Clinical Excellence: Chronic fatique syndrome/myalgic encephalomyelitis (or encephalopathy): Diagnosis and management of CFS/ME in adults and children. NHS National Institute for Health and Clinical Excellence, 7. 2007; NICE clinical guideline 53; 2007. 
2. Prins JB, van der Meer JW, Bleijenberg G: Chronic fatigue syndrome. Lancet 2006, 367:346-355.

3. Collin SM, Crawley E, May MT, Sterne JAC, Hollingworth W: The impact of CFS/ME on employment and productivity in the UK: a cross-sectional study based on the CFS/ME national outcomes database. BMC Health Serv Res 2011, 11:217.

4. Assefi NP, Coy TV, Uslan D, Smith RW, Buchwald D: Financial, occupational, and personal consequences of disability in patients with chronic fatigue syndrome and fibromylagia compared to other fatiguing conditions. J Rheumatol 2011, 30(4):804-808.

5. Hannon K, Peters S, Fisher L, Riste L, Wearden A, Lovell K, Turner P, Leech Y, Chew-Graham C: Developing resources to support the diagnosis and management of Chronic Fatigue Syndrome/Myalgic Encephalitis (CFS/ME) in primary care. A qualitative study. BMC Fam Pract 2012, 13:93.

6. Drachler ML, Leite JCC, Hooper L, Hong CS, Pheby D, Nacul L, Lacerda E, Campion P, Killett A, McArthur M, Poland F: The expressed needs of people with Chronic Fatigue Syndrome/Myalgic Encephalomyelitis: a systematic review. BMC Public Health 2009, 9:458.

7. Chew-Graham CA, Cahill G, Dowrick C, Wearden A, Peters S: Using multiple sources of knowledge to reach clinical understanding of chronic fatigue syndrome. Ann Fam Med 2008, 6(4):340-348.

8. A report of the CFS/ME working group: Report to the Chief Medical Officer of an independent working group. London:The Stationery Office: Department of Health; 2002.

9. Afari N, Buchwald D: Chronic fatigue syndrome: a review. Am J Psychiatry 2003, 160:221-236.

10. Wearden AJ, Dowrick C, Chew-Graham C, Bentall RP, Morriss RK, Riste L, Richardson G, Lovell K, Dunn G: Nurse led, home based self help treatment for patients in primary care with chronic fatigue syndrome: randomised controlled trial. Br Med J 2010, 340:1777.

11. Action for M.E: Severely neglected - M.E. in the UK, 2001; 2012. [online] Source available from: www.afme.org.uk.

12. Stenhoff A, Sadreddini S, Peters S, Wearden A: Understanding medical students' views of chronic fatigue syndrome: a qualitative study. J Health Psychol 2013, 0(0):1-12.

13. Lester $\mathrm{H}$, Hannon $\mathrm{K}$, Campbell S: Identifying unintended consequences of quality indicators: a qualitative study. BMJ Qual Saf 2011, 20(12):1057-1061.

14. Lester $\mathrm{H}$, Campbell S: Developing quality and outcomes framework indicators and the concept of 'QOFability'. Qual Prim Care 2010, 18(2):103-109.

15. Larun L, Malterud K: Identity and coping experiences in chronic fatigue syndrome: a synthesis of qualitative studies. Patient Educ Couns 2007. 10.1016/.jpec.2007.06.008

16. Anderson VR, Jason LA, Hlavaty LE, Porter N, Cudia J: A review and meta synthesis of qualitative studies on myalgic encephalomyelitis/chronic fatigue syndrome. Patient Educ Couns 2012, 86(2):147-155.

17. Britten N, Campbell R, Pope C, Donovan J, Morgan M, Pill R: Using meta ethnography to synthesise qualitative research: a worked example. $J$ Health Serv Res Policy 2002, 7(4):209-215.

18. Noblit GW, Hare RD: Meta-Ethnography: synthesizing qualitative studies. London: Sage Publications; 1988.

19. Asbring P, Narvanen A: Ideal versus reality: physicians perspectives on patients with chronic fatigue syndrome (CFS) and fibromyalgia. Soc Sci Med 2003, 57:711-720

20. Ax S, Greff VH, Jines D: Chronic fatigue syndrome: sufferer's evaluation of medical support. J R Soc Med 1997, 90:250-254.

21. Banks J, Prior L: Doing things with illness. The micro politics of the CFS clinic. Soc Sci Med 2001, 52:11-23.

22. Bayliss K, Riste L, Fisher L, Wearden A, Peters S, Lovell K, Chew-Graham C Diagnosis and management of chronic fatigue syndrome/myalgic encephalitis in black and minority ethnic people: a qualitative study. Prim Health Care Res Dev 2013, 23:1-13.

23. Chew-Graham C, Dixon R, Shaw JW, Smyth N, Lovell K, Peters S: Practice Nurses' views of their role in the management of chronic fatigue syndrome/myalagic encephalitis: a qualitative study. BMC Nursing 2009, 8:2.

24. Chew-Graham C, Dowrick C, Wearden A, Richardson V, Peters S: Making the diagnosis of chronic fatigue syndrome/myalgic encephalitis in primary care: a qualitative study. BMC Fam Pract 2010, 11:16

25. Chew-Graham C, Brooks J, Wearden A, Dowrick C, Peters S: Factors influencing engagement of patients in a novel intervention for CFS/ME: a qualitative study. Prim Health Care Res Dev 2011, 12:112-122.
26. Clarke JN: The search for legitimacy and the "Expertization" of the Lay person. Soc Work Health Care 2000, 30(3):73-93.

27. Dickson A, Knussen C, Flowers P: Stigma and the delegitimisation experience: an interpretative phenomenological analysis of people living with chronic fatigue syndrome. Psychol Health 2007, 22(7):851-867.

28. Edwards CR, Thompson AR, Blair A: An 'Overwhelming Illness': women's experiences of learning to live with chronic fatigue syndrome/myalgic encephalomyelitis. J Health Psychol 2007, 12:203.

29. Gilje AM, Soderlund A, Malterud K: Obstructions for quality care experienced by patients with chronic fatigue syndrome (CFS)- A case study. Patient Educ Couns 2008, 73:36-41.

30. Guise J, McVittie C, McKinlay A: A discourse analytic study of ME/CFS (Chronic Fatigue Syndrome) suffers' experiences of interactions with doctors. J Health Psychol 2010, 15:426.

31. Horton SMC, Poland F, Kale S, Drachler ML, Leite JCC, MCArthur MA Campion PD, Pheby D, Nacul L: Chronic fatigue syndrome/myalgic encephalomyelitis (CFS/ME) in adults: a qualitative study of perspectives from professional practice. BMC Fam Pract 2010, 11:89.

32. Horton-Salway M: Bio-psycho-social reasoning in GP's case narratives: the discursive construction of ME patients' identities health. Health 2002, 6(4):401.

33. McDermott C, Lynch J, Leydon GM: Patients' hopes and expectations of a specialist chronic fatigue syndrome/ME service: a qualitative study. BMC Fam Pract 2011, 28:572-578.

34. Peters S, Wearden A, Morriss R, Dowrick C, Lovell K, Brooks J, Cahill G, Chew-Graham C: Challenges of nurse delivery of psychological interventions for long-term conditions in primary care: a qualitative exploration of the case of chronic fatigue syndrome/myalgic encephalitis. BMC Implementation Sci 2011, 6:32.

35. Raine R, Carter S, Sensky T, Black N: General practitioners' perceptions of chronic fatigue syndrome and beliefs about its management, compared with irritable bowel syndrome: qualitative study. BMJ 2004, 328:1354.

36. Schoofs N, Bambini D, Ronning P, Bielack E, Woehl J: Death of a lifestyle. The effects of social support and healthcare support on the quality of life of persons with fibromyalgia and/or chronic fatigue syndrome. Orthop Nurs 2004, 23(6):364-374.

37. Woodward RV, Broom DH, Legge DG: Diagnosis in chronic illness: disabling or enabling- the case of chronic fatigue syndrome. $J R$ Soc Med 1995, 88:325-329.

38. Critical Appraisal Skills Programme (CASP). Qualitative research checklist. http:// www.casp-uk.net/wp-content/uploads/2011/11/CASP_Qualitative_Appraisal_ Checklist_14oct10.pdf.

39. Elliot $\mathrm{H}$ : Use of formal and informal care among people with prolonged fatigue: a review of the literature. Br J Gen Pract 1999, 49(439):131-134.

40. Thomas MA, Smith AP: Primary healthcare provision and chronic fatigue syndrome: a survey of patients' and general practitioners' beliefs. BMC Fam Pract 2005, 6:49.

41. Bowen J, Pheby D, Charlett A, McNulty C: Chronic fatigue syndrome: a survey of GPs' attitudes and knowledge. Fam Pract 2005, 22(4):389-393.

42. Huibers MJH, Wessely S: The act of diagnosis: pros and cons of labelling chronic fatigue syndrome. Psychol Med 2006, 36(7):895-900.

43. Van Hoof $\mathrm{E}:$ The doctor-patient relationship in chronic fatigue syndrome: survey of patient perspectives. Qual Prim Care 2009, 17(4):263-270.

44. Hak T, Campion P: Achieving a patient-centred consultation by giving feedback in its early phases. Postgrad Med J 1999, 75:405-409.

45. Litva A, Peters S: Exploring barriers to teaching behavioural and social sciences in medical education. Med Educ 2008, 42:309-314.

46. Shattock L, Williamson H, Caldwell K, Anderson K, Peters S: 'They've just got symptoms without science': medical trainees' acquisition of negative attitudes towards patients with medically unexplained symptoms. Patient Educ Couns 2013, 91:249-254.

47. Creed F, Guthrie E, Fink P, Henningsen P, Rief W, Sharpe M, White P: Is there a better term than "Medically unexplained symptoms"? J Psychosom Res 2009, 68(1):5-8.

\section{doi:10.1186/1471-2296-15-44}

Cite this article as: Bayliss et al: Overcoming the barriers to the diagnosis and management of chronic fatigue syndrome/ME in primary care: a meta synthesis of qualitative studies. BMC Family Practice 2014 15:44 